\title{
First results and perspectives of KM3NeT/ARCA
}

\section{R. Coniglione* for the KM3NeT collaboration}

Istituto Nazionale Fisica Nucleare - Laboratori Nazionali del Sud Via S. Sofia 62, Catania, 95123 Italy

E-mail: coniglionedlns.infn.it

The KM3NeT Collaboration started the construction of a research infrastructure hosting a nextgeneration underwater neutrino observatory in the Mediterranean Sea. Within KM3NeT, the ARCA telescope, currently being constructed at the Italian site, will be devoted to the observation and measurement of high-energy cosmic neutrinos. The first two Detection Units of the telescope were operated for several months collecting and transmitting data. From the analyzed data valuable information that validates the detector technology and confirms the unique properties of the Optical Modules, the optical sensors of KM3NeT, has been extracted. Moreover, the new discovery fluxes estimated for the full detector presented in this work confirm that ARCA is an optimal detector to look for Galactic neutrino sources.

Neutrino Oscillation Workshop (NOW2018)

9 - 16 September, 2018

Rosa Marina (Ostuni, Brindisi, Italy)

${ }^{*}$ Speaker. 


\section{Introduction}

The existence of high energy neutrinos from outside our Solar System is nowadays a reality. Despite the recent discovery of a neutrino source by IceCube [1, 2], the origin of the bulk of the observed neutrinos of astrophysical origin is not yet known. Moreover, after the recent Gravitational Wave discoveries is more and more evident that only a joint effort with other multi-messenger observatories (gamma-rays, X-ray, optical, radio) can contribute to solve the puzzle on the mechanisms at work in extremely energetic cosmic objects.

The KM3NeT collaboration is now in the construction phase of two underwater neutrino detectors in the Mediterranean Sea. The physics goal is twofold. With the ORCA (Oscillation Research with Cosmic in the Abyss) detector, that is in construction offshore Toulon, studies on the fundamental neutrino properties will be carried out measuring the atmospheric neutrino flux. The detector geometry has been then optimized to measure neutrino of few GeV. The ARCA (Astroparticle Research with Cosmics in the Abyss) detector, that is in construction offshore the Sicilian coast, will measure and observe neutrinos of astrophysical origin. The geometry of the ARCA neutrino detector has then been optimized for the detection of neutrinos from $10^{2} \mathrm{GeV}$ to $10^{8} \mathrm{GeV}$. ARCA will reach the same size of IceCube providing a complementary field of view and a better angular resolution. ORCA and ARCA will share the same technology for the construction and deployment of the main components of the detectors.

\section{The detector, first results and detector performance}

Each one of the KM3NeT detectors consist of a three dimensional grid of optical sensors. The optical sensors, the Digital Optical Modules (DOMs), are attached to vertical structures, called Detection Units (DUs). The DUs are flexible structures anchored to the sea floor and kept vertical by submerged buoys. The DUs are connected to shore by means of a sea floor network of electrooptical cables. An array of 115 DUs will constitute a detector building block.

The ARCA detector will comprise two building blocks with DUs spaced on average by about $90 \mathrm{~m}$ for a total volume of about $1 \mathrm{~km}^{3}$. It will be installed $80 \mathrm{~km}$ off-shore the Sicilian coast in front of Capo Passero, Italy, at a depth of 3500m. Each DU hosts 18 DOMs suspended along two parallel ropes and vertically spaced by $36 \mathrm{~m}$. The connection of the DOMs to the base of the DU is assured by a flexible oil-filled plastic tube that contains 30 optical fibers for data transport and two copper wires for electrical power. For deployment, a detection unit is wrapped on a spherical frame which is deposited on the seabed and then unfurled in a rotating upwards movement.

Each DOM is a 17-inch diameter pressure-resistant glass sphere housing 31 3-inch photomultiplier tubes (PMTs) together with read out electronics and low power circuits providing highvoltage. [4]. The lower hemisphere of each DOM contains 19 PMTs, which are thus downwardlooking, whereas the other 12 PMTs look upwards. The front-end electronics amplify the PMT signals and transform them into digital time-over-threshold information that is fed into the readout via optical fibres [5]. All PMT signals are sent to shore, where event candidates are selected by online filters running on a computer farm. The DOM also contains three calibration sensors: a LED nano-beacon, that can illuminate the DOMs located vertically above, for time calibration, a compass and tiltmeter for orientation calibration and an acoustic piezo sensor for position calibration. 


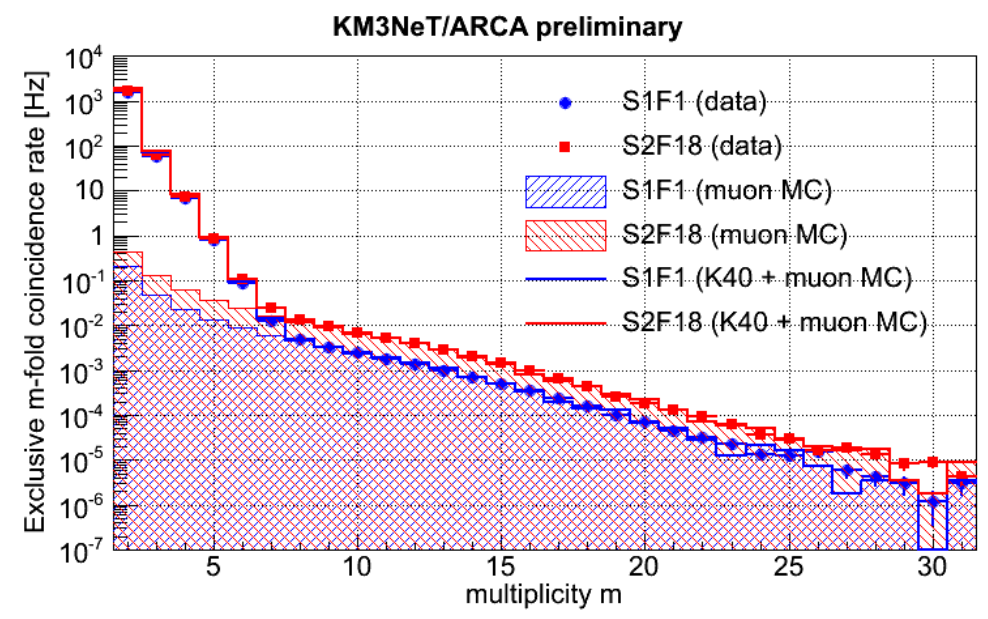

Figure 1: Coincidence rate as a function of the multiplicity for two ARCA DOMs: the lowest DOM of DU1 (S1F1) and the highest DOM of DU2 (S2F18). MC simulations of ${ }^{40} K$ and atmospheric muons are also shown.

The first DU of the ARCA detector was deployed in December 2015 and two further in May 2016. One of the latter was recovered for inspection in July 2016. The DUs were connected to the submarine network and started data taking immediately. Due to electrical problems in the submarine network, the operation of ARCA2 is on hold since April 2017 and is planned to be resumed in early 2019. From the data collected during the functioning of the two ARCA DUs an analysis considering a DOM as a single detector has started. Being the KM3NeT optical sensor a multi-PMT device it is possible to measure the rate of events with multiplicity $m$, where $m$ is the number of PMTs with one or more hits within a time window of 25ns. As shown in Fig.1, where the coincidence rate as a function of the PMT multiplicity for two ARCA DOMs is reported, the coincidence rate for high number of PMT multiplicity $(m>8)$ is due to the atmospheric muons passing near the DOMs while the rate at low multiplicity $(m<6)$ is originated by the genuine coincidences of photons emitted during the $\beta$ decay of ${ }^{40} \mathrm{~K}$ present in salt water. The integrals for $m>8$ is reported for each DOM of the two DUs as a function of the their depth in Fig. 2. The expected dependence of the rate versus depth reproduces the depth dependence of the atmospheric muons as proved by the MonteCarlo based on Mupage (red dashed line in Fig. 2) that is superimposed to the data corrected for the PMT efficiencies estimated with ${ }^{40} \mathrm{~K}$ calibration [6] (green symbols).

The ARCA detector performance for neutrino fluxes from full sky (diffuse flux), from a selected region near the Galactic Center and from two Galactic sources have been reported in the KM3NeT Letter of Intent (LoI) [7]. More recently the event reconstruction algorithms and analysis methods have been improved significantly and more precise $\gamma$-ray observations have also allowed for updated neutrino flux predictions from Galactic sources. An extended set of potential neutrino sources has been investigated. Results are presented in Fig. 3 where the ratio of discovery potential at $3 \sigma$ and the expected neutrino flux is reported as a function of the observation time for the most intense Galactic sources. The most intense sources, the SNR RXJ1713 and the Vela Jr, can be discovered at 3 sigma level in about 6 years. If for these two sources a stacked analysis is carried 


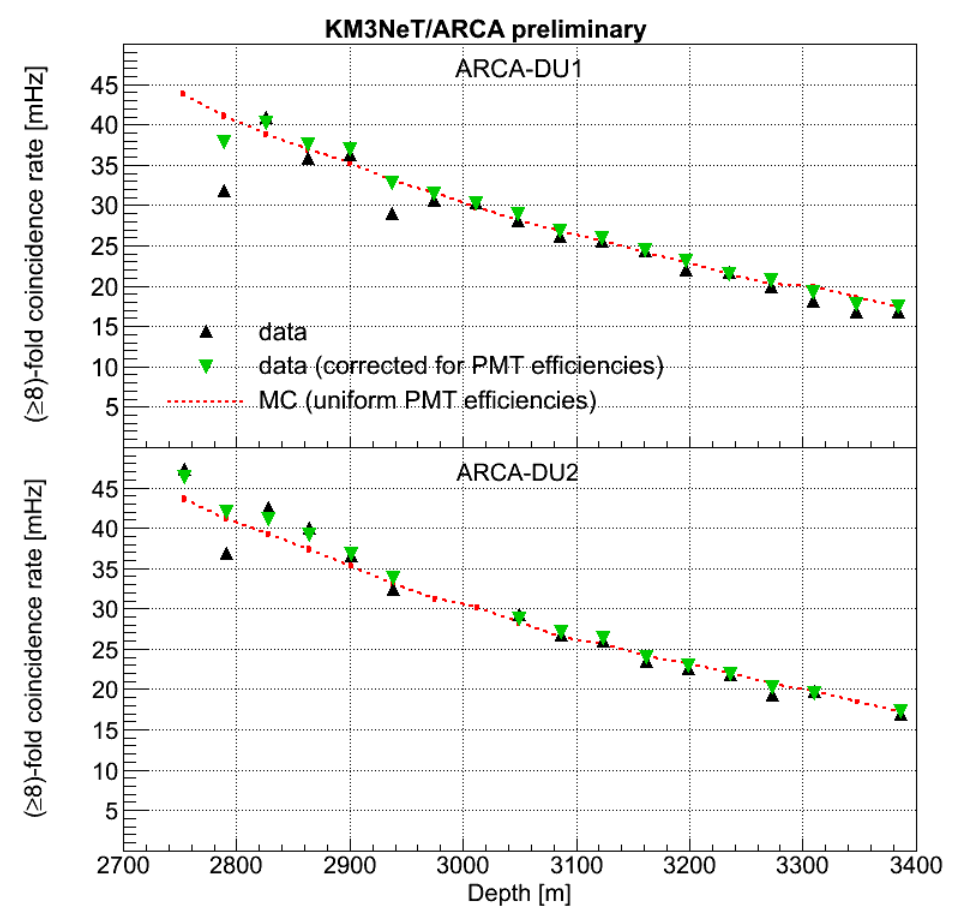

Figure 2: Coincidence rate for PMT multiplicity higher than 8 as a function of the depth (black symbols). Green symbols represent the data corrected for the PMT efficiency. The simulated atmospheric muon flux, based on MUPAGE, is also reported (dashed red line).

out the discovery can be achieved in about 3 years as shown in Fig.4. Full details on the analysis are reported in [8].

\section{Summary}

In this work the status of the KM3NeT/ARCA detector, the main technical components of the detector and performance for Galactic neutrino sources have been briefly presented. The results from the first two DUs deployed show that the innovative optical sensor, developed inside the collaboration, is able to act as a single detector discriminating hits originated by atmospheric muons from background hits. Moreover, new expectation of the discovery potential for Galactic sources shows that in few years a detection at 3 sigma level can be achieved for several sources.

\section{References}

[1] Aartsen M. G. et al. (IceCube Coll.), 2018 Science 361 147-151.

[2] IceCube coll. et al., 2018 Science 361146.

[3] de Wolf E. et al., for the KM3NeT Coll. 2013 Nucl. Inst. Meth. A725 241.

[4] S. Aiello et al., KM3NeT Coll. 2018 JINST 13 P05035.

[5] Jansweijer P. P. M., Peek H. Z. and de Wolf E. 2013 Nucl. Inst. Meth. A725 488. 


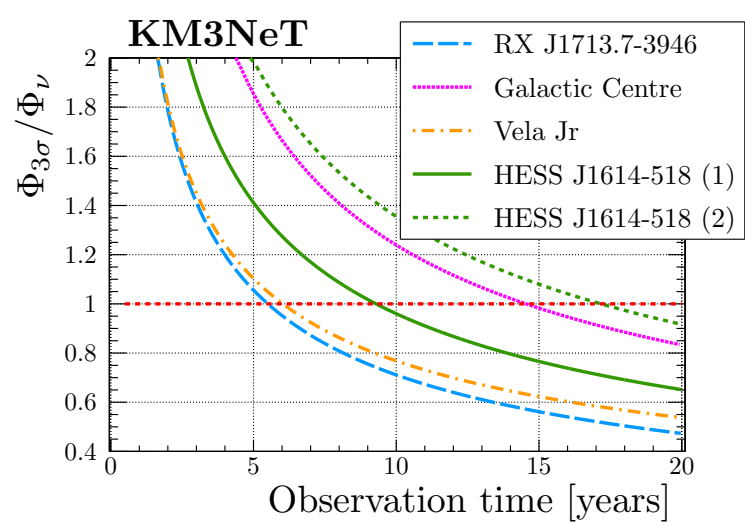

Figure 3: Ratio of the discovery potential $\Phi_{3 \sigma}$ to the expected neutrino flux $\Phi_{v}$ as a function of the observation time for the sources indicated in the legend.

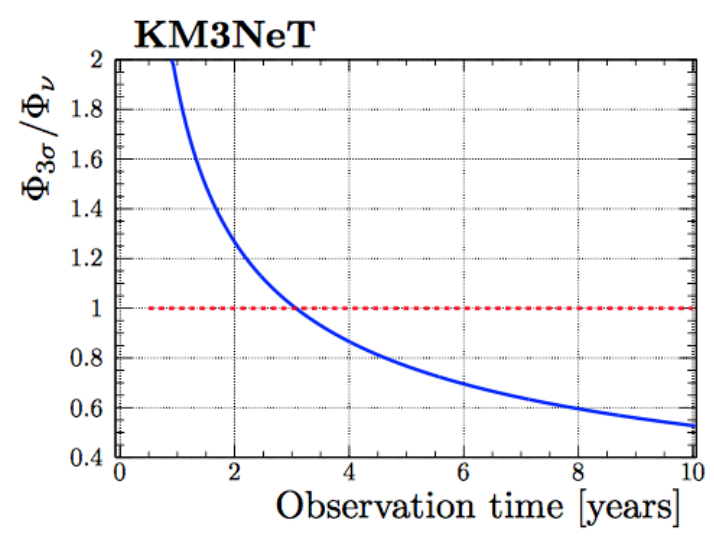

Figure 4: Ratio of the discovery potentials $\Phi_{3 \sigma}$ to the expected neutrino flux $\Phi_{v}$ as a function of the observation time for a stacked analysis including RX J1713.73946 and Vela Jr.

[6] Adrián-Martínez S. et al., KM3NeT Coll. 2016 Eur. Phys. J. C 76:54.

[7] Adrián-Martínez S. et al., KM3NeT Coll. 2016 Journal of Physics G: Nuclear and Particle Physics 43084001.

[8] S. Aiello et al., KM3NeT Coll. 2018 https://arxiv.org/abs/1810.08499. 